\section{SINGLE-CENTRE LONG-TERM SAFETY OUTCOMES OF PATIENTS IMPLANTED WITH SUBCUTANEOUS IMPLANTABLE CARDIOVERTER DEFIBRILLATORS}

Neasa Starr, Sean Esmonde, Nicholas Mc Keag, Engwooi Chew. Belfast Trust Hospitals, Belfast, Northern Ireland

\subsection{6/heartjinl-2021-BCS.96}

Introduction Boston Scientific (location) Emblem $^{\mathrm{TM}}$ subcutaneous implantable cardioverter defibrillator (SICD) is an extrathoracic defibrillator system. Two landmark studies, S-ICD System IDE Clinical Investigation (IDE) and Boston Scientific Post Market S-ICD Registry (Effortless) demonstrated safety profiles similar to transvenous (TV) implantable cardioverter defibrillators (ICDs).

Aim This study aimed to investigate the working safety profile of Boston Scientific SICD Emblem ${ }^{\mathrm{TM}}$ devices implanted in patients attending a hospital trust within the United Kingdom (UK) between February 2015 and 1st March 2020.

Methods This was a single-center, retrospective study that investigated patients followed up at a single UK hospital trust. Data was collected using Boston Scientific Latitude ${ }^{\mathrm{TM}}$ NXT and patient electronic care records.

Results A total of 116 patients with a Boston Scientific Emblem $^{\mathrm{TM}}$ SICD were included in the study. 115 patients had the SICD implanted at the Hospital Trust whilst 1 patient had their implant completed at an external paediatric center. Patient characteristics including SICD indication are summarized in table 1. Over the course of the study follow-up 20 patients experienced at least one event, which were subsequently stratified into appropriate or inappropriate events. 7 (6\%) patients received an appropriate shock for ventricular arrhythmias whilst 7 (6\%) also received an inappropriate shock (IAS). Patients that received inappropriate therapies had a total of 12 shocks delivered overall. Of the total shocks delivered, 7 (58\%) were secondary to non-physiological noise believed to be myopotential oversensing. Of note there were no IAS due to T-wave oversensing. A summary of observed outcomes is presented in table 2. Regarding reintervention, 9 $(7.8 \%)$ patients required a repeat procedure over the course of the study with a total of 12 procedures. Overall $2(1.7 \%)$ patients developed a device infection requiring extraction. Due to symptomatic bradycardia, 2 patients had a TV pacemaker programmed to work in tandem with the SICD. Both patients had bipolar pacing outputs programmed and received no IAS over the course of the study. The complete summary of details regarding the indication for reintervention are included in figure 2. A total of $3(2.6 \%)$ patients died during the study follow-up but none of the deaths were directly attributable to the device or implant procedure. The mean age at death was $67 \pm 13$ yrs, with a mean duration from implant to death of $12.6 \pm 10.8$ months.

Conclusion This study confirms an ongoing working safety profile of implanted SICDs consistent with previous SICD studies. This study however highlights real time data offering further insight into potential issues associated with SICDs. One of the main findings from the study was the fact that of the $6 \%$ of patients that received an IAS $58 \%$ were deemed due to myopotential oversensing. None of the inappropriate shocks detected were due to $\mathrm{T}$ wave oversensing, an issue that appears to have been resolved with the Emblem $^{\mathrm{TM}}$ SMART pass filter. IAS due to myopotential oversensing is therefore an important practical outcome from the study. The impact of myopotential oversensing suggests lead position in relation to the pectoral muscle poses real difficulties and should be taken into consideration as early as vector analysis prior to implant. The importance of detecting non-physiological noise was highlighted by an advisory notification released in December 2020. The advisory highlighted a lead fracture risk at the region of the proximal sensing probe in certain electrodes and generally requires replacement. To detect both non-physiological noise and myopotential interference remote surveillance is

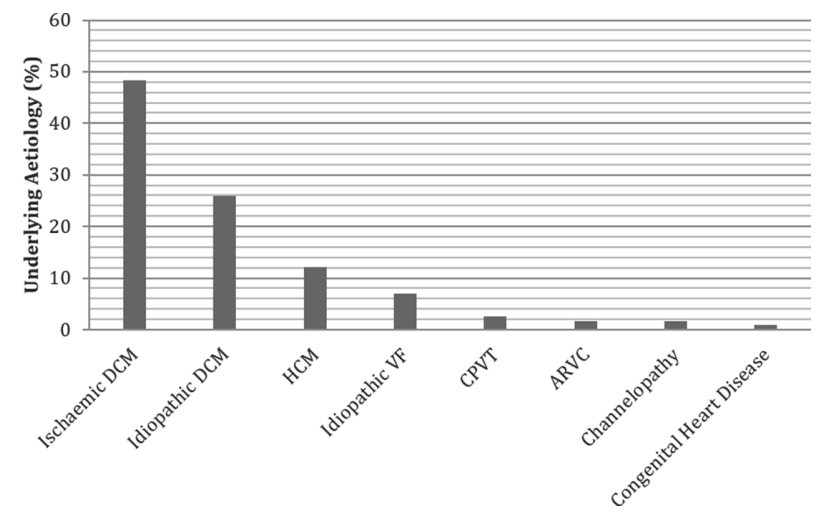

Abstract 97 Figure 1 Underlying a etiology. DCM-dilated cardiomyopathy, HCM- hypertrophic cardiomyopathy, ARVC arrhythmogenic right ventricular cardiomyopathy, VF-ventricular fibrillation, CPVT-catecholaminergic polymorphic ventricular tachycardia

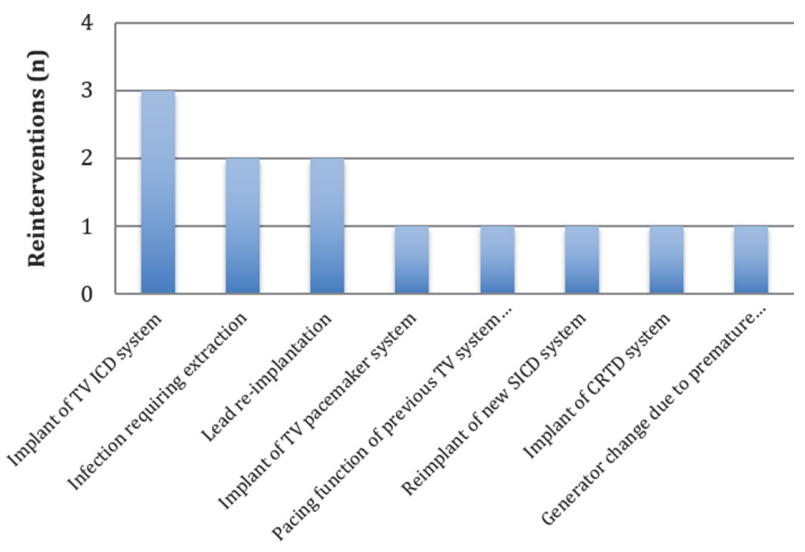

Abstract 97 Figure 2 TV - transvenous, ICD - implantable cardioverter defibrillator, SICD - subcutaneous implantable cardioverter defibrillator, CRTD - cardiac resynchronisation therapy defibrillator

Abstract 97 Table 1 LVEF-left ventricular ejection fraction, DCMdilated ca rdiomyopathy, HCM-hypertrophic cardiomyopathy, ARVC arrhythmogenic right ventricular cardiornyopathy, VF-ventricular fibrillation, CPVT-catecholarninerg ${ }_{i} \mathrm{c}$ polymorphic ventricular tachycardia, AF-Atrial fibrillation

\begin{tabular}{|l|l|}
\hline & All patients (n=116) \\
\hline Total & 116 \\
\hline Age at implant [mean years] & $51 \pm 14$ years \\
\hline Male [n (\%)] & $86(68)$ \\
\hline Female [n (\%)] & $30(32)$ \\
\hline LVEF [mean \%] & 36.7 \\
\hline Primary prevention [n (\%)] & 81 \\
\hline Secondary prevention [n (\%)] & 35 \\
\hline AF [n (\%)] & $28(24)$ \\
\hline $\begin{array}{l}\text { Follow-up post device implantation } \\
\text { [mean months) }\end{array}$ & $24.3 \pm 15.9$ months (range 1.8 - 62.9) \\
\hline
\end{tabular}




\begin{tabular}{|c|c|c|c|}
\hline & Result: & & Result: \\
\hline Number of Total events & 44 & $\begin{array}{l}\text { Number of incorrectly labeled } \\
\text { ventricular events (no therapy } \\
\text { delivered) }\end{array}$ & 12 \\
\hline $\begin{array}{l}\text { Number of patients } \\
\text { (\% of total) }\end{array}$ & $22(18.9)$ & Number of patients (\% of total) & $7(6)$ \\
\hline Appropriate shocks: & & $\begin{array}{l}\mathrm{T} \text { wave oversensing } \text { (event } \\
\text { number with no therapy } \\
\text { delivered) }\end{array}$ & 1 \\
\hline Number of events & 15 & $\begin{array}{l}\text { Non cardiac oversensing (event } \\
\text { number with no therapy } \\
\text { delivered) }\end{array}$ & 11 \\
\hline $\begin{array}{l}\text { Number of patients } \\
\text { (\% of total) }\end{array}$ & $7(6)$ & $\begin{array}{l}\text { SVT (event number with no } \\
\text { therapy delivered) }\end{array}$ & 0 \\
\hline Inappropriate shocks: & & Reintervention: & \\
\hline Number of events & 12 & Number of procedures & 12 \\
\hline $\begin{array}{l}\text { Number of patients } \\
\text { (\% of total) }\end{array}$ & $7(6)$ & Number of patients (\% of total) & $9(7.8)$ \\
\hline
\end{tabular}

therefore an important aspect of care in patients with SICD implants.

Conflict of Interest none

\section{\begin{tabular}{l|l}
98 & SOCIOECONOMIC DEPRIVATION SCORE IMPROVES
\end{tabular} PREDICTION OF AF RECURRENCE AND RE-ADMISSION POST AF ABLATION}

Yee Cheng Lau, Krystalina Yih Tyng Sim, Oana Cimpeanu, Gillian E Marshall, Gareth J Padfield, Gary A Wright, Richard Dobson, Derek T Connelly. NHS Greater Glasgow and Clyde (NHS GGC), Glasgow, UK

\subsection{6/heartjnl-2021-BCS.97}

Background Atrial fibrillation (AF) recurrence post catheter ablation can occur in up to $50 \%$ within 12 months of treatment. Previously, several novel scoring systems have been suggested, inclusive of CHADS2VASc, R2CHADS2, CHADS2 and APPLE score. Nevertheless, prediction of AF recurrence and subsequent readmission remains difficult.

Methods and Results 383 consecutive patients attended the Golden Jubilee National Hospital, Glasgow, to undertake cryoablation for AF. Average age 58 ( \pm 13), 64\% male, BMI 29 $( \pm 4.7), 60 \%$ paroxysmal AF. Degrees of socioeconomic deprivation as per Scottish Index of Multiple Deprivations (SIMD) were recorded in deciles. Patients were followed up to at least 12 months post procedure. After initial 3-month blanking period, symptomatic recurrence of documented AF (not SVT/ Atrial tachycardia/Flutter) requiring admission to hospital were observed in 86 patients $(22.5 \%)$.

Individual CHADS2VASc Score, R2CHADS2 ( $\mathrm{R}$ for renal dysfunction), APPLE Score(one point for Age $>65$ years, Persistent AF, imPaired eGFR ( $<60 \mathrm{ml} / \mathrm{min} / 1.73 \mathrm{~m} 2)$, Left atrial diameter $\geq 43 \mathrm{~mm}$, left ventricular Ejection fraction $<50 \%$ ) were recorded and compared to Scottish Index of Multiple Deprivations (SIMD) using statistical methods to assess for AUC as per ROC Curve.In the current cohort of patients, previous scoring systems CHA2DS2-VASc score (AUC 0.5337 (95\% CI $0.4336-0.6338, \mathrm{p}=0.287)$, R2CHADS2 score (AUC 0.5202 (95\% CI $0.4201-0.6203, \mathrm{p}=0.874)$ and APPLE score (AUC0.5523 (95\% CI 0.4522 - 0.524, p = 0.049)) demonstrated lower predictive values, while SIMD demonstrating better prediction of $\mathrm{AF}$ recurrence requiring readmission (AUC 0.6145 (95\% CI 0.5144 - 0.7146 , $\mathrm{p}=0.001)$ ). SIMD also demonstrated to better predicts recurrence of subjective AF recurrence (AUC $0.6096,0.5095$ $0.7097, \mathrm{p}<0.001)$. Addition of SIMD into CHADS2VASc,
R2CHADS2 or APPLE Score through various modeling did not improve predictive value of AF recurrence or readmission.

Conclusion Scottish Index of Multiple Deprivation is superior to the CHA2DS2-VASc, R2CHADS2 and APPLE scores for prediction of readmission and recurrence of symptomatic AF after AF cryoablation.

Conflict of Interest No

\section{PULMONARY VEIN ISOLATION FOR ATRIAL FIBRILLATION: ICE 'BLOCK' VS RING OF FIRE}

${ }^{1}$ Ashwin Reddy, ${ }^{2}$ Sarah Nethercott, ${ }^{1}$ Bharat Khialani, ${ }^{3}$ Munmohan Virdee. ${ }^{1}$ Papworth Hospital, Cambridge, UK; ${ }^{2}$ Addenbrookes Hospital

\subsection{6/heartjnl-2021-BCS.98}

Background Over the last 20 years various techniques have been developed striving for safer and more durable pulmonary vein isolation (PVI). The popularity and uptake of 'single-shot' strategies, which by their nature are simpler and quicker, has risen in line with the growing worldwide prevalence of $\mathrm{AF}$ and the concomitant requirement to simplify and streamline AF ablation service delivery. The most commonly used singleshot techniques are pulmonary vein ablation catheter (PVAC) and cryoballoon. Head-to-head assessment of outcomes of new-generation cryoballoon vs PVAC gold has never before been performed.

Objective Evaluate the safety and efficacy of PVAC gold vs cryoballoon in an unselected population undergoing de-novo ablation for persistent or paroxysmal atrial fibrillation (AF) at Royal Papworth Hospital (RPH).

Method Retrospective, single-centre study of consecutive AF ablations at RPH over a one year period. Demographic, procedural and outcome data were analysed. Complications were defined as any adverse procedure-related event. Success was defined as freedom from symptoms or demonstrable arrhythmia after 12 months following an initial 3-month blanking period. The consultant performing the procedure determined the ablation method used. Cases performed using point-bypoint techniques or AcQMap were excluded from analysis.

Analysis of parametric continuous data were performed using Student's t-tests, whilst categorical data were compared using the $\chi 2$ test. A two-tailed probability level of $<0.05$ was considered significant. Paroxysmal and persistent AF groups were examined separately due to the well-recognised differences in long-term outcome between the two entities.

Results Over the study period 329 first-time PVI procedures were performed. 131 (39.8\%) were performed using cryoballoon (106 [78.6\%] of which were for pAF) and $75(22.8 \%)$ using PVAC (62 [82.7\%] for pAF). The average age was 60.4 \pm 10 years and $69.6 \%$ of patients were male. There was no significant difference in age, sex, left atrial diameter or cardiovascular co-morbidity profile between different ablation technique groups (table 1). Procedure times were similar between interventions (127 vs 117 mins, $\mathrm{p}=0.79$ ). Acute vein isolation was achieved in $96.9 \%$ of cryoballoon patients and $98.7 \%$ of PVAC patients $(p=0.88) .12$-month freedom from symptomatic AF was not significantly different between cryoballoon and PVAC (75.7 vs $78.6 \%, p=0.99$ for paroxysmal AF; $75.0 \%$ vs $83.3 \%, \mathrm{p}=0.80$ for persistent $\mathrm{AF}$ ) (figure 1).

Overall complication rate was $1.9 \%$, and similar between groups (table 2). A pseudoaneurysm occurred following a 